ACCEPTED MANUSCRIPT

\title{
Mechanical exfoliation and layer number identification of single crystal monoclinic $\mathrm{CrCl}<\mathrm{sub}>3</$ sub $>$
}

To cite this article before publication: Shafaq Kazim et al 2020 Nanotechnology in press https://doi.org/10.1088/1361-6528/ab7de6

\section{Manuscript version: Accepted Manuscript}

Accepted Manuscript is "the version of the article accepted for publication including all changes made as a result of the peer review process, and which may also include the addition to the article by IOP Publishing of a header, an article ID, a cover sheet and/or an 'Accepted

Manuscript' watermark, but excluding any other editing, typesetting or other changes made by IOP Publishing and/or its licensors"

This Accepted Manuscript is @ 2020 IOP Publishing Ltd.

During the embargo period (the 12 month period from the publication of the Version of Record of this article), the Accepted Manuscript is fully protected by copyright and cannot be reused or reposted elsewhere.

As the Version of Record of this article is going to be / has been published on a subscription basis, this Accepted Manuscript is available for reuse under a CC BY-NC-ND 3.0 licence after the 12 month embargo period.

After the embargo period, everyone is permitted to use copy and redistribute this article for non-commercial purposes only, provided that they adhere to all the terms of the licence https://creativecommons.org/licences/by-nc-nd/3.0

Although reasonable endeavours have been taken to obtain all necessary permissions from third parties to include their copyrighted content within this article, their full citation and copyright line may not be present in this Accepted Manuscript version. Before using any content from this article, please refer to the Version of Record on IOPscience once published for full citation and copyright details, as permissions will likely be required. All third party content is fully copyright protected, unless specifically stated otherwise in the figure caption in the Version of Record.

View the article online for updates and enhancements. 
Keywords: two-dimensional materials, ferromagnetism, $\mathrm{CrX}_{3}$, mechanical exfoliation, crystal growth, atomic force microscopy, and Raman spectroscopy.

\title{
Mechanical exfoliation and layer number identification of single crystal monoclinic $\mathrm{CrCl}_{3}$
}

\author{
S Kazim ${ }^{1}$, M Alì ${ }^{2}$, S Palleschi ${ }^{2,4}$, G D'Olimpio ${ }^{2}$, DMastrippolito ${ }^{2}$, A \\ Politano $^{2}$, R Gunnella ${ }^{1}$, A Di Cicco ${ }^{1}$, M Renzelli ${ }^{4}$, G Moccia $^{4}$, O A \\ Cacioppo $^{4}$, R Alfonsetti ${ }^{4}$, J Strychalska-Nowak ${ }^{5}$, T Klimczuk ${ }^{5}$, R J \\ Cava $^{6}$, L Ottaviano $^{2,3}$ \\ ${ }^{1}$ School of Science and Technology Physics division, Università di Camerino, Italy \\ ${ }^{2}$ Department of Physical and Chemical Sciences (DSFC) Università dell'Aquila, \\ L'Aquila, Italy \\ ${ }^{3}$ CNR-SPIN uos L'Aquila, L'Aquila \\ ${ }^{4}$ Lfoundry, Avezzano (AQ), Italy \\ ${ }^{5}$ Faculty of Applied Physics and Mathematics Gdansk University of Technology, \\ Gdansk, Poland \\ ${ }^{6}$ Department of Chemistry Princeton University, Princeton, NJ 08544, USA \\ E-mail: luca.ottaviano@aquila.infn.it
}

Abstract. After the recent finding that $\mathrm{CrI}_{3}$, displays ferromagnetic order down to its monolayer, extensive studies have followed to pursue new two-dimensional (2D) magnetic materials. In this article, we report on the growth of single crystal $\mathrm{CrCl}_{3}$ in the layered monoclinic phase. The system after mechanical exfoliation exhibits stability in ambient air (the degradation occurs on a time scale at least four orders of magnitude longer than is observed for $\mathrm{CrI}_{3}$ ). By means of mechanical cleavage and atomic force microscopy (AFM) combined with optical identification, we demonstrate the systematic isolation of single and few layer flakes onto $270 \mathrm{~nm}$ and $285 \mathrm{~nm}$ $\mathrm{SiO}_{2} / \mathrm{Si}(100)$ substrates with lateral size larger than graphene flakes isolated with the same method. The layer number identification has been carried with statistically significant data, quantifying the optical contrast as a function of the number of layers for up to six layers. Layer dependent optical contrast data have been fitted within the Fresnel equation formalism determining the real and imaginary part of the wavelength dependent refractive index of the material. A layer dependent (532 $\mathrm{nm}$ ) micro-Raman study has been carried out down to two layers with no detectable spectral shifts as a function of the layer number and with respect to the bulk. 


\section{Introduction}

The recent demonstration of ferromagnetism in 2D van der Waals systems has opened a new vast investigation area in the field of 2D materials, with groundbreaking potential for spintronic applications [1-3]. Besides metallic-conducting $\mathrm{Fe}_{3} \mathrm{GeTe}_{2}$ [4], attention has been largely focused on the family of insulating chromium trihalides $\left(\mathrm{CrX}_{3}: \mathrm{X}=\mathrm{Cl}, \mathrm{Br}, \mathrm{I}\right)$ [5].

This class of materials can be referred to as van der Waals magnets, and has been studied largely because it is a rather peculiar case of magnetic insulators [68]. Also, being van der Waals systems, their magnetic properties have been the subject of specific investigation for their quasi-two-dimensional magnetism [9-11]. In chromium tri-halides, the $\mathrm{Cr}$ atoms sit in a honeycomb planar lattice, each $\mathrm{Cr}$ atom is at the center of an octahedron with six halide atoms. With this given structural unit, the layered structure of this class of materials is formed by try-layers where a plane of $\mathrm{Cr}$ atoms is sandwiched between two halide layers (see Figure 1).

As super exchange is by far more important in the development of magnetic order of metal halides, it has to surpass the strength of the direct exchange[12]. From this point of view, namely the development of the magnetic order in the transition metal tri-halides, the $X$ size plays a very critical role. As, by stepping down in the halogen group (from $\mathrm{Cl}$, to $\mathrm{Br}$, to $\mathrm{I}$ ) the $\mathrm{X}$ atomic size increases, the distance between two $\mathrm{Cr}$ atoms also increases. The larger the $\mathrm{Cr}-\mathrm{Cr}$ distance, the weaker is the expected direct exchange, thus making super exchange dominant in the development of magnetic order [13]. Thus while going from $\mathrm{Cl}$ to $\mathrm{Br}$ to I the super exchange is more important in the development of magnetic order. In addition, by stepping down in the halogen group, spin orbit coupling is supposed to increase the anisotropic behaviour in the materials [14, 15]. Going 2D, several theoretical studies supported the magnetic behaviour of monolayers chromium halides, and for the above rationale the focus has been largely put on $\mathrm{CrI}_{3}$ [16]. Indeed, these theories as already mentioned, have been recently experimentally confirmed on few layer $\mathrm{CrI}_{3}$ [5]. According to these experiments, $\mathrm{CrI}_{3}$ tri-layer and monolayer exhibit ferromagnetic (FM) behaviour, while bi-layer $\mathrm{CrI}_{3}$ is antiferromagnetic [5] (AFM). However, $\mathrm{CrI}_{3}$ is extremely prone to degradation in ambient pressures and temperatures [17] and to make experiments viable, graphene or hexagonal boron nitride $(\mathrm{h}-\mathrm{BN})$ encapsulation is required [5, 17]. The chemical stability of $\mathrm{CrI}_{3}$ is therefore a technological game-stopper.

In comparison to $\mathrm{CrI}_{3}, \mathrm{CrCl}_{3}$ has received relatively little attention. Some theoretical studies were performed on $\mathrm{CrCl}_{3}$ by using different van der Waals density functional theories and Monte Carlo methods [12, 16]. These studies observed a change in FM order on thinning to a few layers. Interestingly, some theoretical works have predicted the magnetic order in monolayers should occur at comparatively higher Curie temperature than bulk. Liu and co-workers predicted FM behaviour below $66 \mathrm{~K}$ for $\mathrm{CrCl}_{3}$ monolayers by Monte Carlo simulation and suggested the 
leverage of the Curie temperature by hole doping [12]. Another Monte Carlo study reported, for monolayer $\mathrm{CrCl}_{3}$, a calculated Curie temperature of $49 \mathrm{~K}$. This calculation predicted that the Curie temperature could be increased even more by applied uniaxial strain [16]. Such predictions, together with the recently reported Giant enhancement of interlayer exchange in an ultra-thin $\mathrm{CrCl}_{3}$ [18] make $\mathrm{CrCl}_{3}$ a promising material for spintronic and sensing nano-devices [3, 19]. Only very recently, notable attention has been moved also to this specific system. Few layer 2D magnetism in $\mathrm{CrCl}_{3}$ has been demonstrated and studied by Klein et al. in [18] and previously by Cai et al. in [20]. These works focused on transport properties. Dhalia Klein and colleagues also reported on the investigation of the magnonmagnon coupling of this system [21] and similarly, the magnon excitation has been investigated in [22].

Any fundamental or technological study on a 2D material starts from a systematic demonstration of the viability of exfoliation and unambiguous layer number identification. To date, although studies on few layer $\mathrm{CrCl}_{3}$ have been already reported $[13,18]$, such a systematic study for this specific material is still lacking.

In this paper, we report on the synthesis of high quality crystalline $\mathrm{CrCl}_{3}$ in the monoclinic phase and its mechanical exfoliation down to the monolayer limit. The mechanical exfoliation has been carried out with the blue tape method, by choosing as dielectric substrate for the optical identification $270 \mathrm{~nm}$ and $285 \mathrm{~nm} \mathrm{SiO} 2 / \mathrm{Si}(100)$ substrates. We demonstrate that the freshly exfoliated flakes are rather inert to ambient air on a time scale of tens of hours. The exfoliation into very large lateral size flakes is rather facile. The optimal contrast for layer number identification is obtained on $285 \mathrm{~nm} \mathrm{SiO}_{2} / \mathrm{Si}(100)$ substrates. The contrast is quantified, through AFM measurements, in terms of layer number up to six layers. Room temperature (RT) layer dependent Raman measurements indicate no appreciable shifts in the Raman spectral modes down to the two-layer limit.

\section{Experimental details}

Single crystals of $\mathrm{CrCl}_{3}$ / were grown via the self-transport technique using commercially available $\mathrm{CrCl}_{3}$ powders. The $\mathrm{CrCl}_{3}$ powder was first submitted to oxygen purification cycles. The purified powder was sealed in a $20 \mathrm{~cm}$ long quartz glass tube and placed in a three-zone furnace with a temperature gradient of approximately $25{ }^{\circ} \mathrm{C}$ between the hot zone and the cold zone. The hot end of the tube was heated to $1000{ }^{\circ} \mathrm{C}$, kept at this temperature for 1 hour and then slowly cooled down to $700{ }^{\circ} \mathrm{C}$ at 3 degree/hours cooling rate. The quartz tube was then held at $700{ }^{\circ} \mathrm{C}$ for 7 days. The as-grown crystals were obtained in a form of shiny, purple flakes with a size of several millimetres (Figure 1). Crystals were stored and manipulated in a glove box with argon atmosphere to avoid oxidation and water contamination. 
$\mathrm{CrCl}_{3}$ few-layer flakes were mechanically exfoliated in the glove box by using the optimized scotch tape method described in ref. [23], using atomically flat $270 \mathrm{~nm}$ and $285 \mathrm{~nm} \mathrm{SiO}_{2} / \mathrm{Si}(100)$. Before the flake transfer, the substrates were pre-cleaned with acetone, isopropanol and distilled water for 10 minutes in ultrasonic bath.

$X$-ray diffraction (XRD) was performed to determine the crystal structure and orientation of the crystals. For the experiments, a crystal plate was laid down on a zero-background holder. The XRD pattern was collected at RT using a Bruker D8 diffractometer with $\mathrm{Cu} \mathrm{K}$ radiation, in the $2 \theta$ range from $10^{\circ}$ to $110^{\circ}$.

Raman spectroscopy was performed with a HORIBA IHR320 micro-Raman Scattering system equipped with an optical Microscope model Olympus BXF41 (with 5X, 20X, 50X, 100X objectives). The Raman spectrometer was operated at $532 \mathrm{~nm}$ (diode laser).

The optical identification of exfoliated $\mathrm{CrCl}_{3}$ flakes was carried out with an Olympus BH2-UMA optical microscope equipped with 5x, 20x, 50x, 100x objectives (numerical aperture $=0.9$ ) under broadband illumination with an unfiltered halogen light (as in ref. [23]). The system operates with a JVC digital camera model TK-C1380. The experimental optical contrast (O.C.) values were calculated as discussed in ref. [23] and compared with theoretical predictions based on the Fresnel formalism (as discussed in the SI):

$$
\text { O.C }=\frac{I_{\text {substrate }}-I_{\text {flake }}}{I_{\text {substrate }}+I_{\text {flake }}}
$$

AFM images were acquired in tapping mode by using a D5000 Veeco system with P-doped n-type Si cantilever (resonance frequency $=75 \mathrm{kHz}$ ).

\section{Results and Discussion}

The crystalline quality of the as grown $\mathrm{CrCl}_{3}$ samples was checked with XRD and Raman spectroscopy. Figure 1 (a) presents a RT XRD pattern of a single crystal of $\mathrm{CrCl}_{3}$. In order to observe low intensity reflections, data are plotted with a squareroot intensity scale. Experimental data points are shown with open black circles and the calculated pattern is represented with a green line. Below $\mathrm{RT} \mathrm{CrCl}_{3}$ undergoes a structural phase transition [24]. In particular, detailed structural studies by McGuire, et al. reveal that above $240 \mathrm{~K} \mathrm{CrCl}_{3}$ adopts the monoclinic $C 2 / \mathrm{m}$ space group and rhombohedral $R-3$ space group below $130 \mathrm{~K}$. Between $130 \mathrm{~K}$ and $240 \mathrm{~K}$ both phases are present [13].

Our XRD experiment was performed at RT, and hence the starting model for the LeBail refinement assumes the monoclinic $C 2 / m$ space group with lattice parameters $\mathrm{a}=5.959 \AA, \mathrm{b}=10.321 \AA, \mathrm{c}=6.114 \AA$ and $\beta=108.5^{\circ}$ [24]. Since a flake-like single crystal was used for the XRD experiment, only $(00 l)$ reflections are observed. Therefore, we refined the spacing $(d)$ between the $(00 l)$ layers from our data. From this XRD analysis the $d$ value obtained is $5.801 \AA$, which is in excellent agreement 

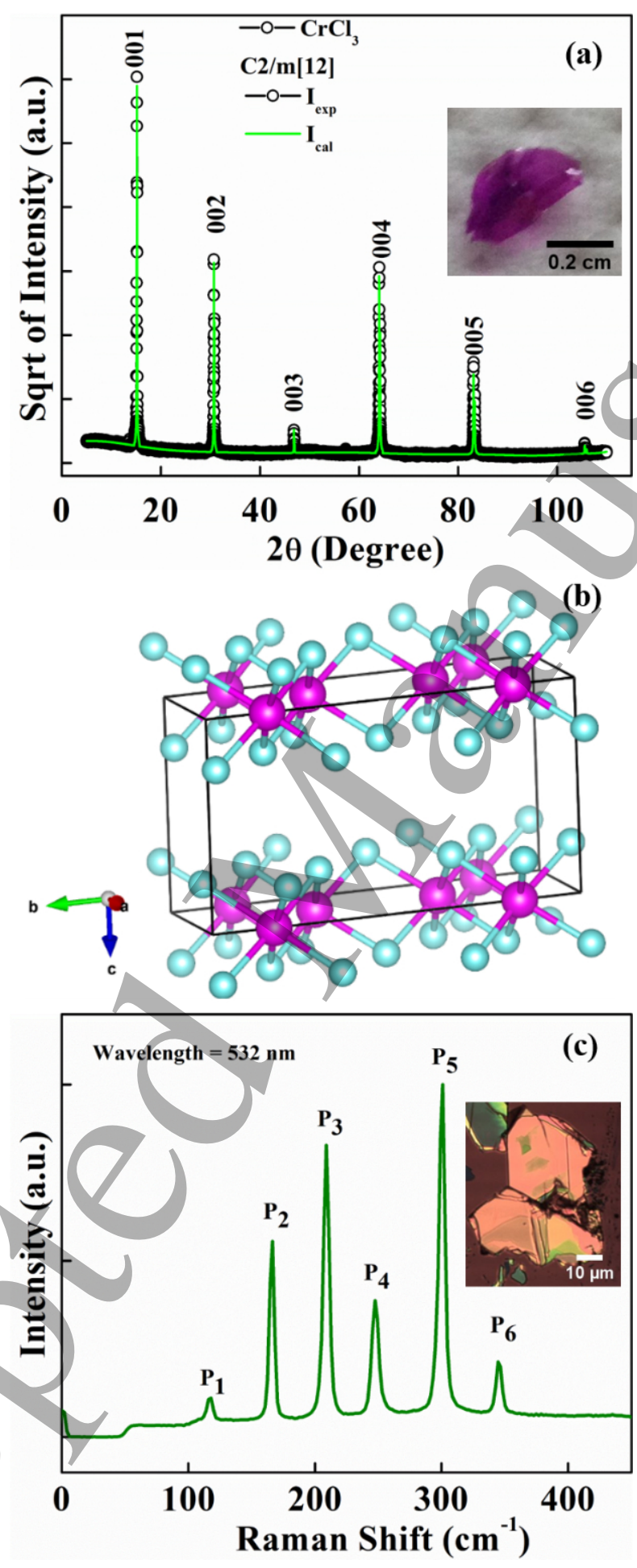

Figure 1: (a) X-ray diffraction pattern of a $\mathrm{CrCl}_{3}$ flake used for the mechanical exfoliation. The image of a $\mathrm{CrCl}_{3}$ single crystal is shown in the inset. Black circles indicate the experimental data and the green line represent the LeBail fit assuming a monoclinic structure. (b) Conventional and primitive unit cell of monoclinic $\mathrm{CrCl}_{3}$ with $\mathrm{Cr}$ (pink) and $\mathrm{Cl}$ (light blue) atoms (see ref. [24]); (c) RT $532 \mathrm{~nm}$ Raman spectrum of bulk $\mathrm{CrCl}_{3}$, (data collected from the flake reported in the inset). 
with the $d=c \cdot \sin \beta=5.798 \AA$ value calculated based on the $c$ and $\beta$ angle values reported previously [24]. The monoclinic crystal structure is reported in Figure 1 (b).

In Figure 1 (c) a typical RT Raman spectrum of a thick flake (inset of Figure 1 (c)) is reported. The spectrum shows six Raman resonant modes at $116.2 \mathrm{~cm}^{-1}, 166.2$ $\mathrm{cm}^{-1}, 208.6 \mathrm{~cm}^{-1}, 247.0 \mathrm{~cm}^{-1}, 300.7 \mathrm{~cm}^{-1}$, and $345.6 \mathrm{~cm}^{-1}$ respectively. Data are fully consistent with those recently reported in ref. [25], where extensive work has been carried out as a function of temperature and light polarisation. These resonances are assigned to six $A_{g}$ modes $\left(A_{g}(1-6)\right.$ ) (those are almost energy degenerate with six $B_{g}$ modes) according to lattice dynamic calculation [26].

Hereafter we focus on the stability of $\mathrm{CrCl}_{3}$ and its mechanical exfoliation. Firstly, we checked its ageing in ambient air. The results of such investigation are summarised in Figure 2 (a)-(d) (AFM investigation) and Figure 3 (a)-(d) (Optical Microscopy investigation).

In Figure 2 (a)-(d) it is reported a few layer flake with the left portion (as commented in the following) exhibiting a stacking height distance from the substrate (3.7 $\mathrm{nm}=$ consistent with the presence of a monolayer $2 \mathrm{D}$ material (as discussed in [27] (see the lower left inset line profile in panel (a)). Upon exposure to ambient air we do not observe any remarkable evidence of surface degradation. In Particular, there is no evidence of etching at the flakes' edges as reported for $\mathrm{CrI}_{3}$ [3]. Another systematic observation is an apparent improvement of the AFM image resolution with time. It reaches its maximum already after 36 hours air exposure (panel (c)). We assign the smearing of the morphological features observed in Figure 2 (a) to meniscus effects with water. The presence of water at the surface and the interface with the $\mathrm{SiO}_{2}$ substrate in few layer mechanically exfoliated materials has been clearly evidenced in recent works on $\mathrm{MoS}_{2}$ [27]. We therefore observe, via the improvement of the AFM resolution a process of de-wetting of the water film both from the substrate and on the flake surface. As this de-wetting is observed both on the flake's and substrate's surfaces we cannot conclude that there are clear indications of ageing and morphological surface degradation of the exfoliated flakes. The de-wetting is confirmed on an larger length scale in Figure 3 where we report the optical image of a thick flake as taken from the glove box and after $24 \mathrm{~h}$ (b), $48 \mathrm{~h}$ (c), and $72 \mathrm{~h}$ (d) of exposure to ambient air respectively. The Figure 3 clearly shows the size increase of a circular spot at the boundary of the green and red areas that mimic on a larger scale the coalescence of water droplets on the flakes surface. Although we cannot exclude chemical contaminations at the surface, however, in any case, if compared with the $\mathrm{CrI}_{3}$ case that exhibits evident degradation on a time scale of tens of seconds [17], we can certainly asses that, for $\mathrm{CrCl}_{3}$ the timescale for surface contamination lies in the range of hundreds of thousand of seconds, therefore $\mathrm{CrCl}_{3}$ is, in terms of time stability, four orders of magnitude better that $\mathrm{CrI}_{3}$.

The exfoliation and layer number identification of $\mathrm{CrCl}_{3}$ is carried out with combined optical microscopy and AFM. The results are summarised in Figure 4 and Figure 5 and in the supporting AFM evidences of Fig. S1 and S2. 


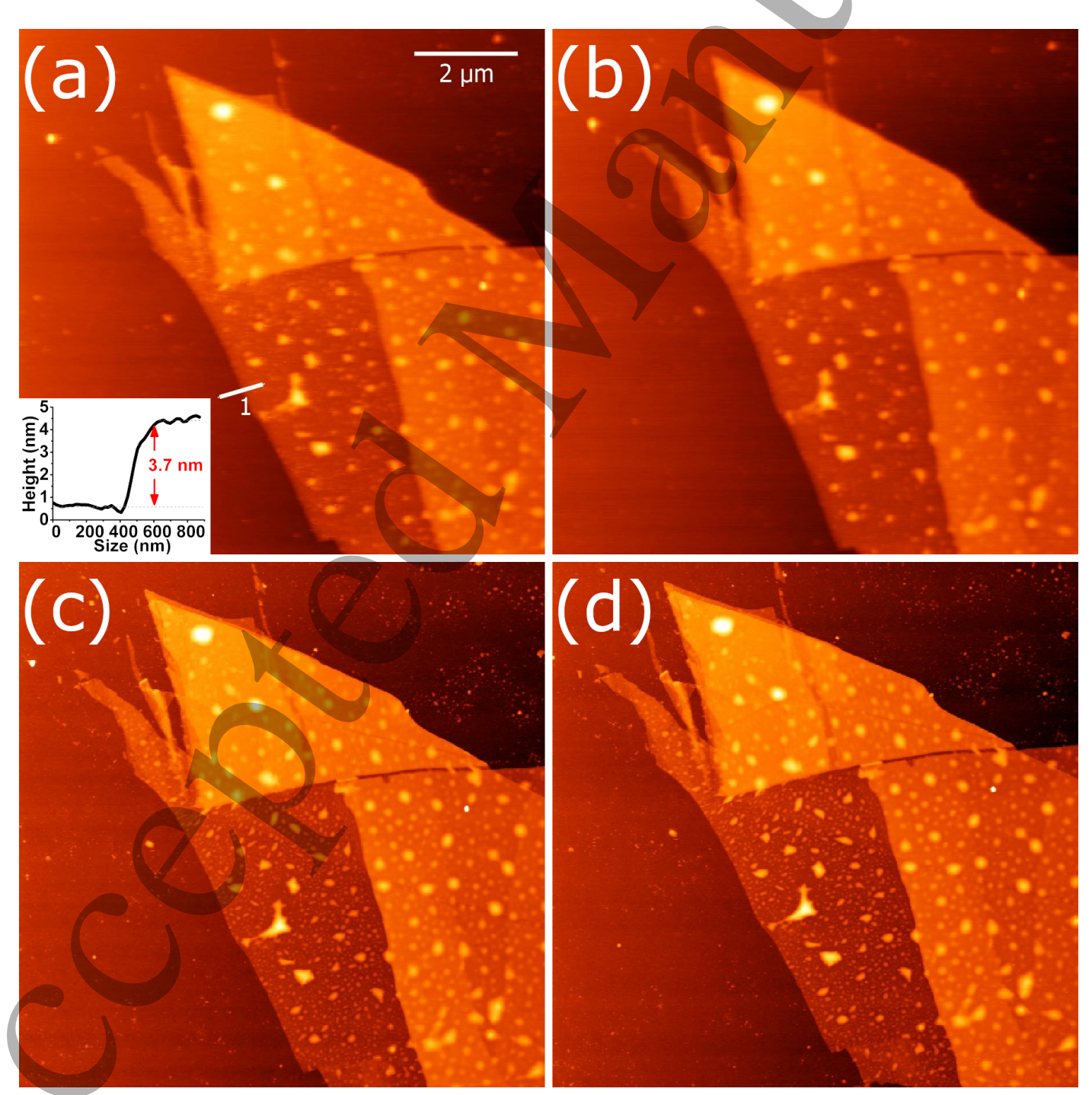

Figure 2: Ageing analysis of a $\mathrm{CrCl}_{3}$ few layer flake in ambient conditions investigated with Atomic Force Microscopy: a) image of the freshly exfoliated flake and (b), (c), and (d) after 24, 48, and 72 hours respectively. average lateral size compared to other standard 2D materials like graphene or $\mathrm{MoS}_{2}$. Flakes have a typical lateral size ranging from $30 \mu \mathrm{m}$ to more than $100 \mu \mathrm{m}$. This is experimentally in line with the observation that, for $\mathrm{CrCl}_{3}$, the cleavage energy has been estimated to be $0.13 \mathrm{~J} / \mathrm{m}^{2}$, namely the lowest for $\mathrm{CrX}_{3}$ layered [12] and accounting for just one third of the corresponding cleavage energy of graphite $(0.37$ $\left.\mathrm{J} / \mathrm{m}^{2}\right)[28]$.

In Figure 4 (a), we show a nice representative case with an exfoliated large flake exhibiting a layered structure where the optical contrast can be calculated as 0.02 , $0.06,0.12$ and 0.17 for the regions labelled as $1 \mathrm{~L}, 2 \mathrm{~L}, 3 \mathrm{~L}$ and $5 \mathrm{~L}$ respectively. For a corresponding unambiguous assignment of the layer numbers, the same flake has 

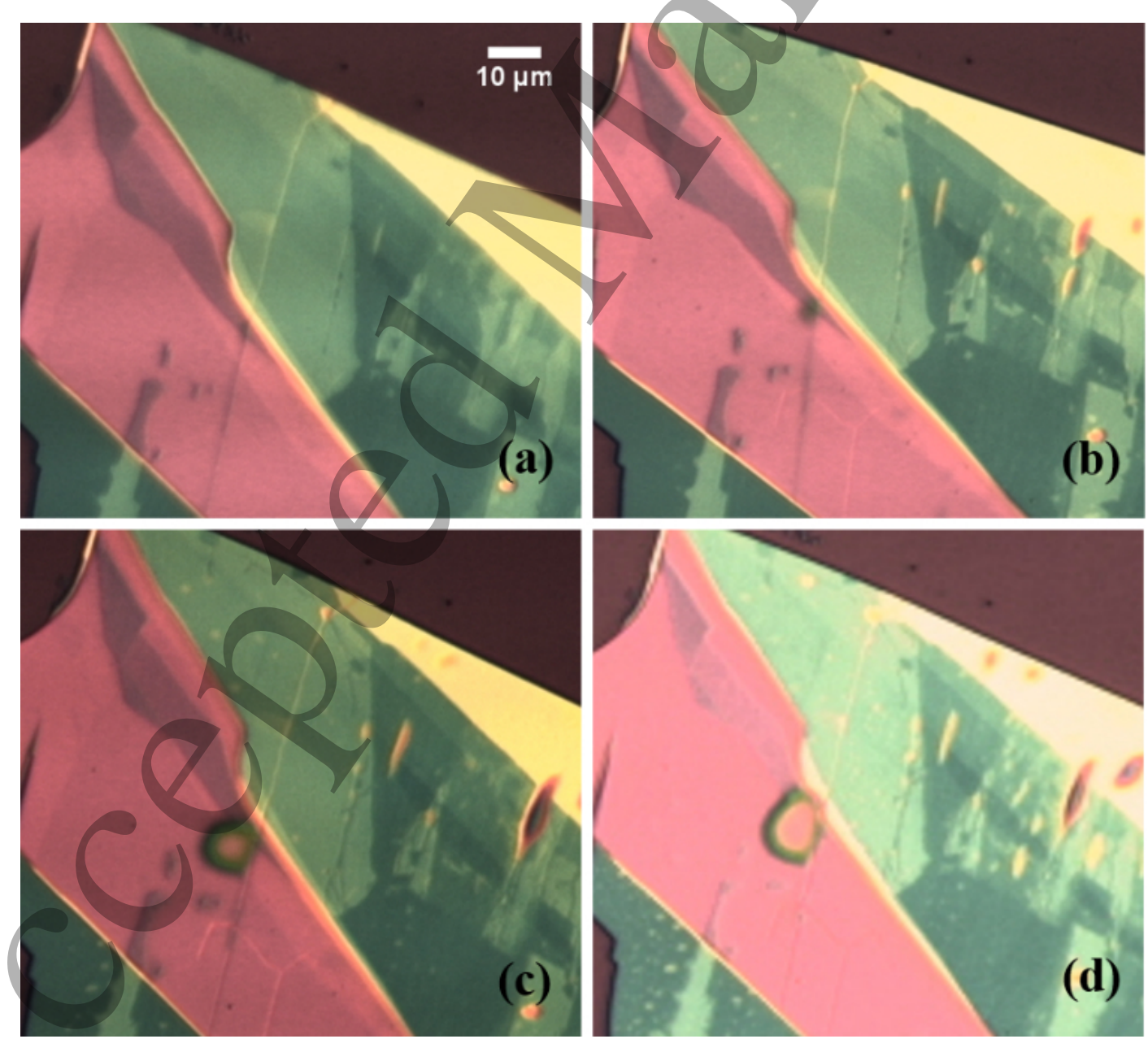

Figure 3: Degradation analysis by optical microscopy of a $\mathrm{CrCl}_{3}$ thick flake in ambient conditions: a) image of the freshly exfoliated flake and (b), (c), and (d) after 24,48 , and 72 hours respectively. substrate) of the portion of the flake showing the faintest optical contrast in panel (a) has been evaluated as $2.3 \mathrm{~nm}$ (see Figure 4 (b)). This value is, again, in the range of the typical stacking distance observed in tapping mode AFM for mechanically exfoliated single layers of layered materials in the presence of substrate contaminants and of an absorbed film of water [23]. For such reason we hereafter call $\mathrm{CrCl}_{3}$ flakes exhibiting a similar optical contrast and a similar AFM stacking height from the substrate "monolayers". The region labelled in Figure 4 (a) as $2 \mathrm{~L}$ has, as determined by AFM (see Figure 4 (d) inset), a stacking distance from the 1L region of $0.7 \pm 0.1$ $\mathrm{nm}$. This value, within the experimental accuracy of AFM, is fully consistent with a single layer stacking distance in $\mathrm{CrCl}_{3}$. Similarly, the 3L region has been verified to stack twice the $\mathrm{c}$ axis distance from the $1 \mathrm{~L}$ one, and a similar AFM check has been performed for the $5 \mathrm{~L}$ region, that we therefore associate consistently with the evidence of stacking of five layers of $\mathrm{CrCl}_{3}$. 
For optical identification, several samples with mechanically exfoliated flakes have been transferred on to $270 \mathrm{~nm} \mathrm{SiO} 2 / \mathrm{Si}$ (100) Figure 5 (a)-(d) and $285 \mathrm{~nm}$ $\mathrm{SiO}_{2} / \mathrm{Si}$ (100) Figure 5 (e)-(h). In each panel of Figure 5, we have reported (from left to right ((a) to (d) and (e) to (h) for $270 \mathrm{~nm}$ and $285 \mathrm{~nm} \mathrm{SiO} 2 / \mathrm{Si}$ (100) respectively) typical optical microscopy images of exfoliated few layer flakes of $\mathrm{CrCl}_{3}$ exhibiting an increasing optical contrast (as calculated from data in the framed areas according to Eq. 1) with respect to the bare substrate. Each panel represents a single value of optical contrast for a particular flake but the final optical contrast value is calculated from the average value of multiple flakes for each layer number. The AFM images corresponding to Figure 5 (b)-(h) are reported in the SI Fig.S1-S3. The values of the optical contrast found in our work are consistent with those measured for $\mathrm{CrI}_{3}[5$,
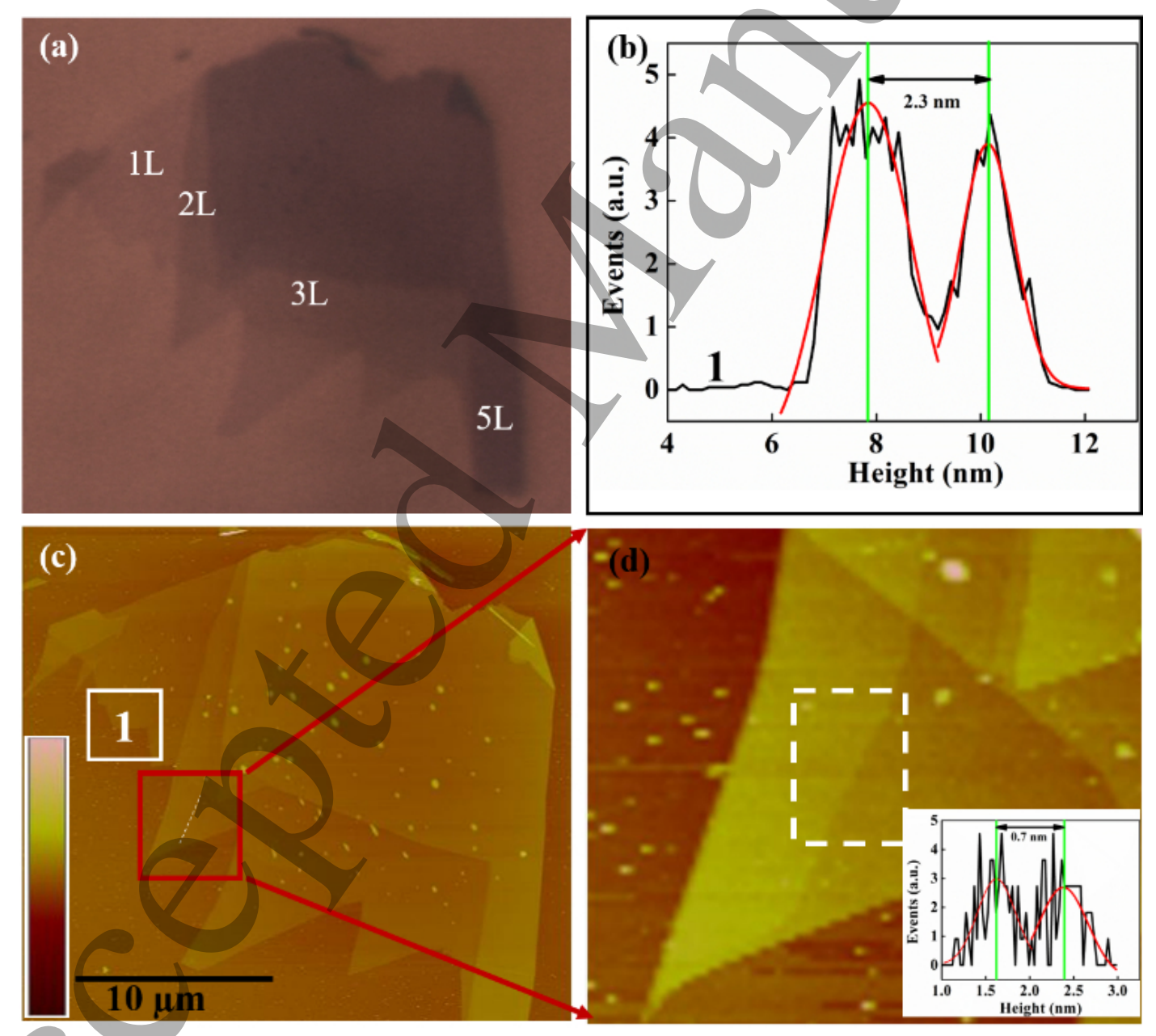

Figure 4: (a) Optical microscopy image, and (c) corresponding AFM image of a mechanically exfoliated few layer flake of $\mathrm{CrCl}_{3}$ on $285 \mathrm{~nm} \mathrm{SiO} 2 / \mathrm{Si}$ (100). (b) Height histogram taken from the areas in (c) framed as [1], and (d) Zoom image of the red squared area to evidence, with a height profile, the stacking between two adjacent layers. 
29].

In Figure 5 (i) data accumulate at specific values that are ordered in a stepwise increase and that we assign in increasing order to an increasing number of layers from one to six. We observe a systematic increased contrast for the $270 \mathrm{~nm} \mathrm{SiO} / \mathrm{Si}(100)$. Noteworthy, as displayed in the inset of Figure 5 (i), this corresponds to a notable $20 \%$ $(13 \%)$ relative increase of the contrast for the monolayer (bilayer) flakes. As already stressed in the literature $[23,30]$. The theoretical framework to quantitatively explain the oxide thickness and layer number dependent variation of the optical contrast is given by the Fresnel equations as discussed and reported in SI. Within such model, our experimental data are nicely fitted by the expected theoretical contrast (fitting lines in Figure 5 (i)) once in the model the $\mathrm{SiO}_{2}$ thicknesses parameters and refractive index are fixed and the $\mathrm{CrCl}_{3}$ complex refractive index is adjusted to $(2.20+0.48 i)$ and $(2.24+0.40 i)$ for both the two substrates. We have also verified that the best agreement between experiment and theory is obtained once in the Fresnel equations the wavelength is adjusted at $550 \pm 20 \mathrm{~nm}$.

Finally, in Figure 6 we report the result of a layer dependent Raman study on few-layer flakes of $\mathrm{CrCl}_{3}$ after AFM and optical contrast characterisation. Raw spectra are reported in panel (a) while in panel (b) the same data are plotted after subtraction of the Si background and upon arbitrary normalisation of each spectrum to the Raman features that are detectable up to the bi-layer case (namely the P3 peak at $208.6 \mathrm{~cm}^{-1}$ ). Notably, apart from an obvious lowering of the intensity of the $\mathrm{CrCl}_{3}$ features due to the layer thinning, within the spectral resolution limit of $1 \mathrm{~cm}^{-1}$ of the technique, we do not observe any spectral shift as a function of the number of layers. To compare with the existing literature, as already stressed before, the results related to the bulk phase are in agreement with what reported recently [25] or even in the seminal work of Bermudez [31]. Such results are also in agreement with the frequency shifts theoretically calculated by Avram et al. [26]. In a recent paper [32] a thickness dependent Raman study has been reported on $\mathrm{CVD}$ grown $\mathrm{CrCl}_{3}$, where the bulk phase spectra are related to a thin film and an ultra-thin layer (with non quantitative thickness information) showing the absence of any detectable spectral shifts. Only if temperature and polarisation dependent studies are performed, like in [18] then a minor (within $2 \mathrm{~cm}^{-1}$ ) spectral shift of the resonance at $247 \mathrm{~cm}^{-1}$ is observed in the bulk at temperatures below $240 \mathrm{~K}$, while this occurrence is reported to occur in a much narrow range of $1 \mathrm{~cm}^{-1}$ for $\mathrm{nm}$ thick flakes. To the best of our knowledge, there are so-far no layer dependent Raman studies for exfoliated $\mathrm{CrCl}_{3}$, while, in the case fo $\mathrm{CrI}_{3}$ a careful layer dependent Raman investigation was reported for 2L, 3L, and bulk very recently [33]. In that work it is evidenced how the most remarkable spectral differences are detected with low-temperature $(10 \mathrm{~K})$ polarised micro-Raman set-ups and manifest themselves in significant layer dependent changes of the relative spectral weight rather than in significant frequency shifts. It is evident then, from our layer dependent measurements, that, at variance with other two dimensional materials, like for example graphene [30], or $\mathrm{MoS}_{2}$ 

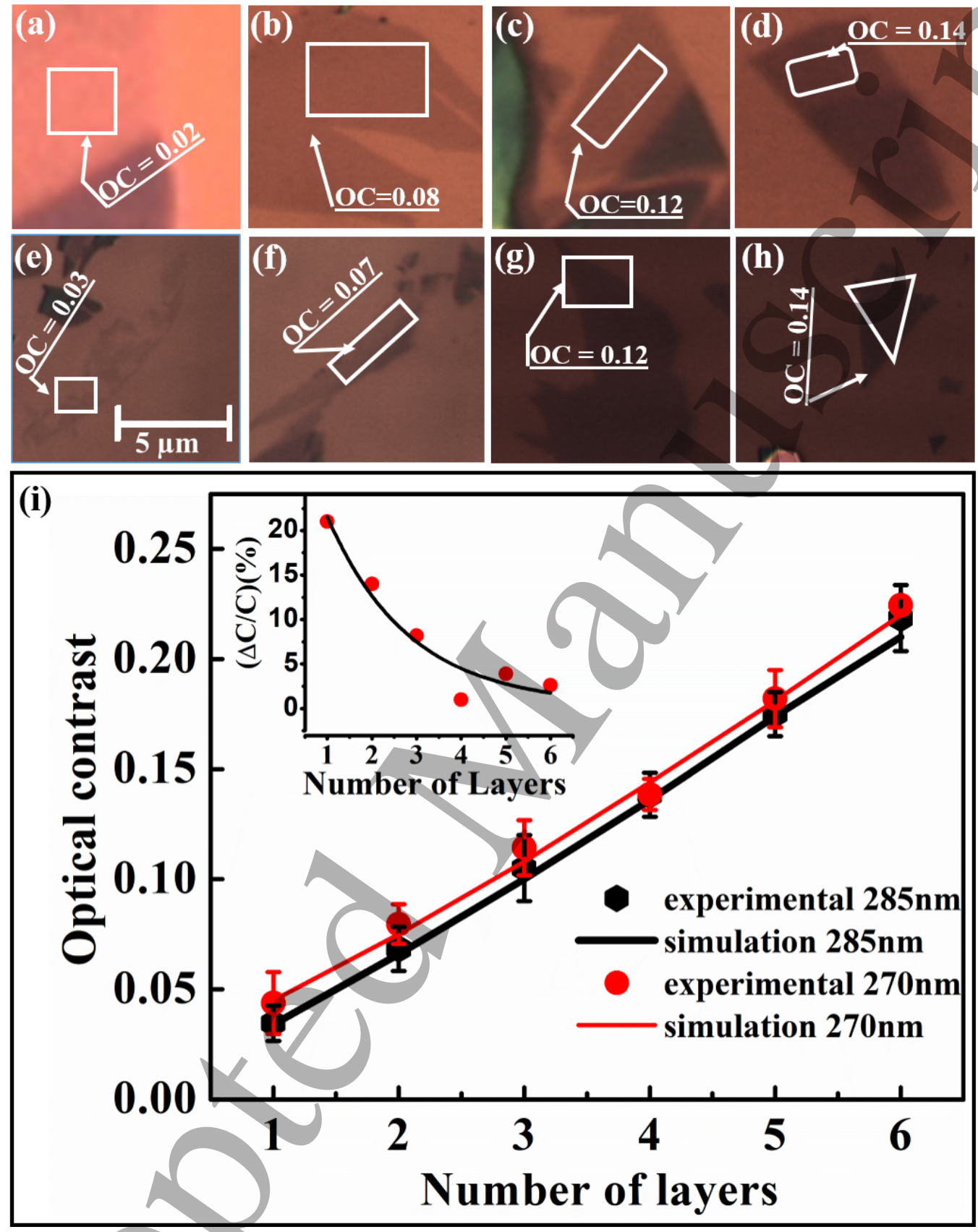

Figure 5: Optical contrast analysis of few-layer $\mathrm{CrCl}_{3}$ flakes: (a-d) represent optical images on a $270 \mathrm{~nm} \mathrm{SiO} 2 / \mathrm{Si}$ substrate and (e-h) on a $285 \mathrm{~nm} \mathrm{SiO} / \mathrm{Si}$ substrate, along with their calculated optical contrasts (measured from data in the framed areas in the figures). (i) The circles and hexagons represent the average value (for each layer number) of the optical contrast on $270 \mathrm{~nm}$ and $285 \mathrm{~nm} \mathrm{SiO} 2 / \mathrm{Si}$ substrates respectively. Experimental data are superimposed with the theoretically calculated contrast based on the Fresnel equations (See SI). The inset in (i) shows the ratio of the measured optical contrast for the two $\mathrm{SiO}_{2}$ substrates as a function of the layer number. 


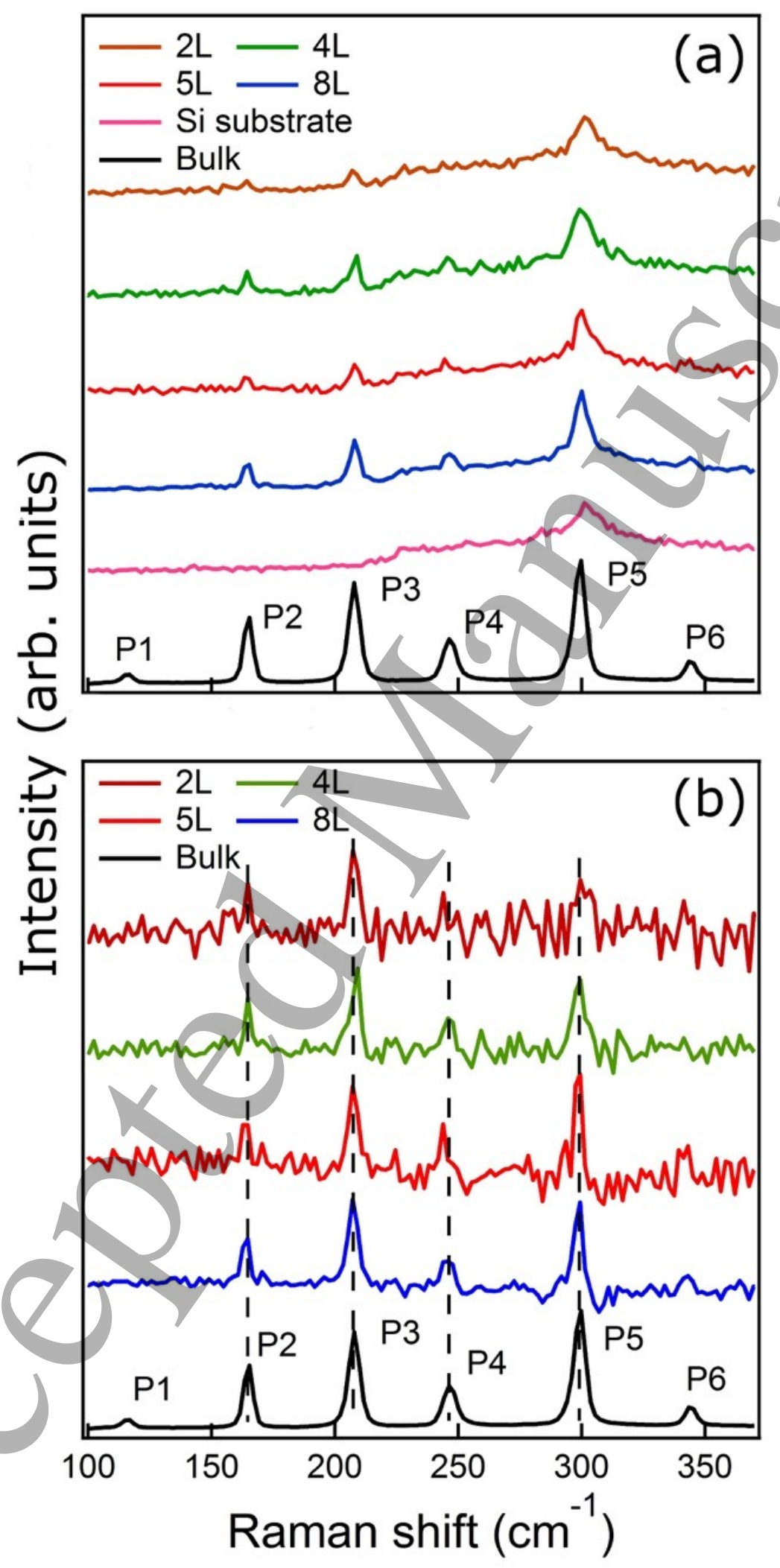

Figure 6: Layer dependent Raman spectra of few-layer (2,4,5, and $8 \mathrm{~L}) \mathrm{CrCl}_{3}$ flakes compared to the spectrum of the bulk phase. (a) raw data, and (b) same corresponding spectra after subtraction of the background from the Si substrate and normalisation to unit of the P3 spectral intensity 
[23], where non polarised RT micro-Raman spectroscopy clearly assign a layer dependent spectral finger print to the 2D material, this is not the case for $\mathrm{CrCl}_{3}$ and likely other $\mathrm{CrX}_{3}$ systems. We ultimately assign this occurrence to the very weak interlayer coupling in these materials. Thus, whether, essentially for such reason their mechanical exfoliation is more easy, on the other hand the individual layers can be considered as already isolated, with little electronic perturbation owed to the confinement in few layers. This is suggested also by the flatness (with dispersion within $0.3-0.4 \mathrm{eV}$ ) of the electronic bands close to the Fermi edge as evidenced in the theoretical calculation of the electronic structure of this system [34].

\section{Conclusions}

In summary, we have demonstrated the systematic exfoliation of monoclinic $\mathrm{CrCl}_{3}$ down to the monolayer limit. In line with theoretical expectations, this 2D material can be exfoliated into large flakes due to its very low cleavage energy. Furthermore, few-layer flakes exposed to ambient air conditions at room temperature exhibit no apparent degradation on a time scale that is four orders of magnitude larger than the one reported for $\mathrm{CrI}_{3}$.

We systematically performed the contrast analysis for two substrates $(270 \mathrm{~nm}$ and $285 \mathrm{~nm} \mathrm{SiO} 2 / \mathrm{Si}(100)$ ) up to six layers and backed the optical microscopy measurements with AFM characterisation and rationalised our findings of the contrast within the Fresnel equation formalism. Micro Raman studies have been carried out on bulk as well as on few-layer $\mathrm{CrCl}_{3}$ at RT. Spectra related to the bulk validated previously reported results [31]. We did not observe any significant layer dependent spectral change down to two layers. Together with recent results reported in the literature focused on transport measurements, this work addressing the viability of the mechanical exfoliation and the the ambient stability of $\mathrm{CrCl}_{3}$ demonstrates the viability of the use of this specific $\mathrm{Cr}$-halide in $2 \mathrm{D}$ spintronic devices.

\section{Acknowledgements}

The research at Princeton University was supported by the ARO MURI on topological insulators grant W911NF-12-1-0461. S. P. and G. D. acknowledge the program PON-2014-2020 "Industrial Doctorates" financed by the European Union. 


\section{REFERENCES}

\section{References}

[1] Klein D R et al. 2018. Probing magnetism in 2D van der Waals crystalline insulators via electron tunneling. Science 360.6394, pp. 1218-1222.

[2] Ghazaryan D et al. 2018. Magnon-assisted tunnelling in van der Waals heterostructures based on $\mathrm{CrBr}_{3}$. Nature Electronics 1.6, pp. 344-349.

[3] Wang Z, Gutiérrez-Lezama I, Ubrig N, Kroner M, Gibertini M, Tanjguchi T, Watanabe K, Imamoğlu A, Giannini E, and Morpurgo A F. 2018. Very large tunneling magnetoresistance in layered magnetic semiconductor $\mathrm{CrI}_{3}$. Nat. Commun. 9.1, p. 2516.

[4] Deng Y, Yu Y, Song Y, Zhang J, Wang N Z, Sun Z, Yi Y, Wu YZ, Wu S, Zhu J, et al. 2018. Gate-tunable room-temperature ferromagnetism in two-dimensional $\mathrm{Fe}_{3} \mathrm{GeTe}_{2}$. Nature 563.7729, pp. 94-99.

[5] Huang B, Clark G, Navarro-Moratalla E, Klein D R, Cheng R, Seyler K L, Zhong D, Schmidgall E, McGuire M A, Cobden D H, et al. 2017. Layer-dependent ferromagnetism in a van der Waals crystal down to the monolayer limit. Nature 546.7657, pp. 270-273.

[6] Dillon J F and Olson C E. 1965. Magnetization, Resonance, and Optical Properties of the Ferromagnet $\mathrm{CrI}_{3}$. Journal of Applied Physics 36.3, pp. 12591260.

[7] Cable J, Wilkinson M, and Wollan E. 1961. Neutron diffraction investigation of antiferromagnetism in $\mathrm{CrCl}_{3}$. Journal of Physics and Chemistry of Solids 19.1, pp. 29-34.

[8] Sandercock J. 1974. A light scattering study of the ferromagnet $\mathrm{CrBr}_{3}$. Solid State Communications 15.10, pp. 1715-1719.

[9] Narath A and Davis H L. 1965. Spin-Wave Analysis of the Sublattice Magnetization Behavior of Antiferromagnetic and Ferromagnetic $\mathrm{CrCl}_{3}$. Phys. Rev. 137 (1A), A163-A178.

[10] Kuhlow B. 1982. Magnetic Ordering in $\mathrm{CrCl}_{3}$ at the Phase Transition. physica status solidi (a) 72.1, pp. 161-168.

[11] Samuelsen E J, Silberglitt R, Shirane G, and Remeika J P. 1971. Spin Waves in Ferromagnetic $\mathrm{CrBr}_{3}$ Studied by Inelastic Neutron Scattering. Phys. Rev. $B 3$ (1), pp. 157-166.

[12] Liu J, Sun Q, Kawazoe Y, and Jena P. 2016. Exfoliating biocompatible ferromagnetic Cr-trihalide monolayers. Phys. Chem. Chem. Phys. 18 (13), pp. 8777-8784.

[13] McGuire M A, Clark G, KC S, Chance W M, Jellison G E, Cooper V R, Xu X, and Sales B C. 2017. Magnetic behavior and spin-lattice coupling in cleavable van der Waals layered $\mathrm{CrCl}_{3}$ crystals. Phys. Rev. Materials 1 (1), p. 014001.

[14] McGuire M A, Dixit H, Cooper V R, and Sales B C. 2015. Coupling of Crystal Structure and Magnetism in the Layered, Ferromagnetic Insulator $\mathrm{CrI}_{3}$. Chemistry of Materials 27.2, pp. 612-620. 
[15] Lado J L and Fernández-Rossier J. 2017. On the origin of magnetic anisotropy in two dimensional $\mathrm{CrI}_{3} .2 \mathrm{D}$ Materials 4.3, p. 035002.

[16] Zhang W.-B, Qu Q, Zhu P, and Lam C.-H. 2015. Robust intrinsic ferromagnetism and half semiconductivity in stable two-dimensional single-layer chromium trihalides. J. Mater. Chem. C 3 (48), pp. 12457-12468.

[17] Shcherbakov D et al. 2018. Raman Spectroscopy, Photocatalytic Degradation, and Stabilization of Atomically Thin Chromium Tri-iodide. Nano Letters 18.7, pp. 4214-4219.

[18] Klein D R et al. 2019. Enhancement of interlayer exchange in an ultrathin twodimensional magnet. Nature Physics 15.12, pp. 1255-1260.

[19] Soumyanarayanan A, Reyren N, Fert A, and Panagopoulos C. 2016. Emergent phenomena induced by spin-orbit coupling at surfaces and interfaces. Nature 539.7630, pp. 509-517.

[20] Cai $\mathrm{X}$ et al. 2019. Atomically Thin $\mathrm{CrCl}_{3}$ : An In-Plane Layered Antiferromagnetic Insulator. Nano Letters 19.6, pp. 3993-3998.

[21] MacNeill D, Hou J T, Klein D R, Zhang P, Jarillo-Herrero P, and Liu L. 2019. Gigahertz Frequency Antiferromagnetic Resonance and Strong MagnonMagnon Coupling in the Layered Crystal $\mathrm{CrCl}_{3}$. Phys. Rev. Lett. 123 (4), p. 047204.

[22] Kim H H et al. 2019. Evolution of interlayer and intralayer magnetism in three atomically thin chromium trihalides. Proceedings of the National Academy of Sciences 116.23, pp. 11131-11136.

[23] Ottaviano L et al. 2017. Mechanical exfoliation and layer number identification of $\mathrm{MoS}_{2}$ revisited. 2D Materials 4.4, p. 045013.

[24] Morosin B and Narath A. 1964. X-Ray Diffraction and Nuclear Quadrupole Resonance Studies of Chromium Trichloride. J. Chem. Phys. 40.7, pp. 1958-1967.

[25] Glamazda A, Lemmens P, Do S.-H, Kwon Y S, and Choi K.-Y. 2017. Relation between Kitaev magnetism and structure in $\alpha-\mathrm{RuCl}_{3}$. Phys. Rev. B 95 (17), p. 174429.

[26] Avram C, Gruia A, Brik M, and Barb A. 2015. Calculations of the electronic levels, spin-Hamiltonian parameters and vibrational spectra for the $\mathrm{CrCl}_{3}$ layered crystals. Physica B: Condensed Matter 478, pp. 31-35.

[27] Palleschi $S$ et al. 2019. On the role of nano-confined water at the $2 \mathrm{D} / \mathrm{SiO}_{2}$ interface in layer number engineering of exfoliated $\mathrm{MoS}_{2}$ via thermal annealing. 2D Materials.

[28] Wang W, Dai S, Li X, Yang J, Srolovitz D J, and Zheng Q. 2015. Measurement of the cleavage energy of graphite. Nature communications 6, p. 7853.

[29] Seyler K L, Zhong D, Klein D R, Gao S, Zhang X, Huang B, Navarro-Moratalla E, Yang L, Cobden D H, McGuire M A, et al. 2018. Ligand-field helical luminescence in a 2D ferromagnetic insulator. Nature Physics 14.3, pp. 277-281. 


\section{REFERENCES}

[30] Marco P D, Nardone M, Vitto A D, Alessandri M, Santucci S, and Ottaviano L. 2010. Rapid identification of graphene flakes: alumina does it better. Nanotechnology 21.25, p. 255703.

[31] Bermudez V M. 1976. Unit-cell vibrational spectra of chromium trichoride and chromium tribromide. Solid State Communications 19.8, pp. 693-697.

[32] Grönke $\mathrm{M}$ et al. 2019. Chromium Trihalides $\mathrm{CrX3}(\mathrm{X}=\mathrm{Cl}, \mathrm{Br}, \mathrm{I})$ : Direct Deposition of Micro- and Nanosheets on Substrates by Chemical Vapor Transport. Advanced Materials Interfaces 6.24, p. 1901410.

[33] Guo K, Deng B, Liu Z, Gao C, Shi Z, Bi Lei and Zhang L, Lu Haipeng and Zhou P, Zhang L, Cheng Y, and Peng B. 2019. Layer dependence of stacking order in nonencapsulated few-layer $\mathrm{CrI}_{3}$. Science China Materials, pp. 1-8.

[34] Wang H, Eyert V, and Schwingenschlögl U. 2011. Electronic structure and magnetic ordering of the semiconducting chromium trihalides $\mathrm{CrCl}_{3}, \mathrm{CrBr}_{3}$, and $\mathrm{CrI}_{3}$. Journal of Physics: Condensed Matter 23.11, p. 116003. 